\title{
Retraction
}

\section{Targeting ceramide synthase 6-dependent metastasis-prone phenotype in lung cancer cells}

Motoshi Suzuki, Ke Cao, Seiichi Kato, Yuji Komizu, Naoki Mizutani, Kouji Tanaka, Chinatsu Arima, Mei Chee Tai, Kiyoshi Yanagisawa, Norie Togawa, Takahiro Shiraishi, Noriyasu Usami, Tetsuo Taniguchi, Takayuki Fukui, Kohei Yokoi, Keiko Wakahara, Yoshinori Hasegawa, Yukiko Mizutani, Yasuyuki Igarashi, Jin-ichi Inokuchi, Soichiro Iwaki, Satoshi Fujii, Akira Satou, Yoko Matsumoto, Ryuichi Ueoka, Keiko Tamiya-Koizumi, Takashi Murate, Mitsuhiro Nakamura, Mamoru Kyogashima, and Takashi Takahashi

Original citation: J Clin Invest. 2016;126(1):254-265. https://doi.org/10.1172/JCI79775.

Citation for this retraction: J Clin Invest. 2019;129(11):5050. https://doi.org/10.1172/JCI133806.

At the request of the corresponding author, the JCI is retracting this article. Following an institutional investigative review of errors in the article, it was determined that the data reported in Figure $1 \mathrm{~B}$ were an analysis of E2F1 expression rather than CERS6 expression, as originally reported. The correct data do not show a difference in CERS6 expression with invasion status in human lung adenocarcinomas. In addition, errors were noted in Supplemental Figure 2, Supplemental Figure 3B, and Supplemental Table 1. The institutional review found no evidence of intention to falsify results.

The authors apologize for these errors. 